\title{
BMJ Open Protocol for a systematic review and meta-analysis of long-term neurocognitive outcomes in paediatric traumatic brain injury
}

\author{
Dawn Shu Hui Looi, ${ }^{1}$ Mark Sen Liang Goh (1) , ${ }^{2}$ Sharon Si Min Goh, ${ }^{3}$ Jia Ling Goh, ${ }^{4}$ \\ Rehena Sultana, ${ }^{5}$ Jan Hau Lee, ${ }^{6}$ Shu-Ling Chong (i) ${ }^{7}$
}

To cite: Looi DSH, Goh MSL, Goh SSM, et al. Protocol for a systematic review and meta-analysis of long-term neurocognitive outcomes in paediatric traumatic brain injury. BMJ Open 2020;10:e035513. doi:10.1136/ bmjopen-2019-035513

- Prepublication history and additional material for this paper are available online. To view these files, please visit the journal online (http://dx.doi org/10.1136/bmjopen-2019035513).

Received 04 November 2019 Revised 24 March 2020 Accepted 21 May 2020

Check for updates

(C) Author(s) (or their employer(s)) 2020. Re-use permitted under CC BY-NC. No commercial re-use. See rights and permissions. Published by BMJ.

For numbered affiliations see end of article.

Correspondence to

Dr Shu-Ling Chong;

chong.shu-ling@kkh.com.sg

\section{ABSTRACT}

Introduction Children who suffer from traumatic brain injury (TBI) are at risk of permanent brain damage and developmental deficits. Reports on neurodevelopmental outcomes in paediatric TBI suffer from small sample size and varying outcome definitions in the neurocognitive domains tested. This protocol describes a systematic review and meta-analysis of paediatric TBI in the following key neurocognitive domains: executive function, perceptual-motor function, language, learning and memory, social cognition and complex attention.

Methods A comprehensive search comprising studies from Medline, Cochrane, Embase and PsycINF0 published from 1988 to 2019 will be conducted. We will include studies on children $\leq 18$ years old who suffer from mild, moderate and severe TBI as determined by the Glasgow Coma Scale that report neurocognitive outcomes in domains predetermined by the Diagnostic and Statistical Manual of Mental Disorders fifth edition criteria. Systematic reviews, meta-analyses, randomised controlled trials, case-control, cohort and cross-sectional studies will be included. References from systematic reviews and meta-analyses will be hand-searched for relevant articles. A meta-analysis will be performed and effect sizes will be calculated to summarise the magnitude of change in each neurocognitive domain compared at different timepoints and stratified by severity of TBI. Included studies will be pooled using pooled standardised mean differences with a random effects model to determine an overall effect. In the scenario that we are unable to pool the studies, we will perform a narrative analysis.

Ethics and dissemination Ethics approval is not required for this study. The authors of this study will publish and present the findings in a peer-reviewed journal as well as national and international conferences. The results of this study will provide understanding into the association between different severities of paediatric TBI and long-term neurocognitive outcomes.

PROSPERO registration number CRD42020152680.

\section{INTRODUCTION}

\section{Rationale}

Traumatic brain injury (TBI) is a major public health issue affecting at least 3 million
Strengths and limitations of this study

- This is a comprehensive review of neurocognitive outcomes among children with traumatic brain injuries of all severities.

- We employ neurocognitive domains as defined by the Diagnostic and Statistical Manual of Mental Disorders, fifth Edition.

- Medical Subject Headings terms are dependent on indexing and may not cover all relevant articles.

- Limited number of databases are employed and studies are restricted to those in English.

- Outcomes outside the predetermined domains will not be included.

children worldwide each year, ${ }^{1}$ resulting in death and disability, especially among moderate and severe TBI. Global mortality rates from TBI range from 2.8 to 3.8 per 100000 children each year. ${ }^{1}$ In 2005 , it was estimated that $1.1 \%$ (3.17 million) of the population in the USA, lived with a long-term disability as a result of TBI. ${ }^{2}$

Children affected by TBI may experience a combination of cognitive, behavioural, and emotional sequelae. ${ }^{3}$ While more than $80 \%$ of TBI cases are mild ${ }^{1}$ and mong these few sustain long-term neurocognitive impairments, ${ }^{4}$ children with moderate and severe TBI show deficits that persist past a child's developmental years into adulthood, affecting educational outcomes, employment outcomes, psychosocial functioning and quality of life. ${ }^{4-6}$

Several studies have been published on long-term neurocognitive outcomes in paediatric TBI. ${ }^{7-12}$ However, these reports are highly varied in the neurocognitive domains chosen, number of domains measured and severity of TBI assessed. The most current meta-analysis reviewed data from 1988 to 2007, covering traditional neurocognitive 
domains of general intellectual functioning, attention or executive functioning, memory and visual perception or motor skills. ${ }^{4}$ There has since been an update in neurocognitive domains made to the Diagnostic and Statistical Manual of Mental Disorders fifth edition (DSM-V) criteria. ${ }^{13}$ Until, there has not been a systematic review or meta-analysis performed using this new DSM-V criteria.

\section{Objectives}

We aim to identify and collate existing evidence from 1988 to 2019 and perform a systematic review and metaanalysis of neurocognitive outcomes following paediatric TBI.

The Population, Exposure, Comparator and Outcome (PECO) question format ${ }^{14}$ will be used where we will study children 18 years old and younger who have been exposed to TBI. These patients with TBI with measured neurocognitive outcomes will be compared with either (1) preinjury baseline measurements or (2) healthy controls or orthopaedic injury patients matched for age and gender. The neurocognitive outcomes, used in this study, are chosen with reference to the DSM-V criteria: executive function, perceptual-motor function, language, learning and memory, social cognition and complex attention. ${ }^{13}$ We aim to evaluate changes in neurocognitive outcome over three defined timepoints (time 1: $0-5$ months, time 2: 6-23 months and time 3: $\geq 24$ months). Patients will be stratified by TBI severity. Quantifiable outcome measures will be pooled within each time frame as defined.

\section{METHODS}

We will use the Preferred Reporting Items for Systematic Reviews and Meta-Analyses Protocols (PRISMA) checklist ${ }^{15}$ when writing our report.

\section{Eligibility criteria}

We will include studies that contain all the elements outlined in the PECO format. These studies should involve a paediatric population between 0 and 18 years old. This is consistent with the lower age limit for insurance coverage for children in the United States and has been used to define the paediatric age group in literature. ${ }^{16}$ Should a study contain data of an age range including both children and adults, it will be included if data pertaining to children can be extracted, or if the author contacted provides us with the relevant ageappropriate data. Provided we have sufficient studies, age groups $(0-5$ and $6-18$ years old) will be evaluated using meta-regression. We chose these two age categories because most children would have begun formal education by 6 years old and they have greater utilisation of language and metacognitive skills that separate them from the $0-5$ years age group. ${ }^{4}$ The exposure must be of TBI, and the clinical assessment must include Glasgow Coma Scale (GCS) scores to stratify the severity of TBI intomild (GCS 13-15), moderate (GCS 9-12) and severe (GCS 3-8). ${ }^{3}{ }^{17}$ TBI is defined as 'an alteration in brain function, or other evidence of brain pathology, caused by an external force'. ${ }^{18}$ This includes any loss of consciousness, amnesia, neurological deficit, change in mental status or radiological evidence of brain pathology. ${ }^{18}$ The study must have reported outcomes of at least one of the selected neurocognitive domains. Outcome measures of neurocognition must correspond to the domains and subdomains outlined by DSM-V ${ }^{13}$ as follows: (1) executive function and planning, decision-making, working memory, responding to feedback, inhibition and flexibility, (2) perceptual-motor function and visual perception, visuoconstructional reasoning, perceptual motor coordination, (3) language and object naming, wordfinding, fluency, grammar and syntax, and receptive language, (4) learning and memory and free recall, cued recall, recognition memory, semantic and autobiographical long-term memory and implicit learning, (5) social cognition and recognition of emotions, theory of mind and insight, (6) complex attention and sustained attention, divided attention, selective attention and processing speed. Time point(s) when measurements are taken postinjury must be reported. A measure of comparison must be present-either (1) preinjury baseline measurements of the TBI subjects or (2) test scores of healthy controls or orthopaedic injury patients matched for age and gender. Ideally, preinjury comparisons would account for interindividual variability and form better comparison. It is preferred to include longitudinal studies given our aim to study long-term changes, however, preinjury baseline is not documented in most studies and is mostly reported only for mild TBI in the setting of sports concussions. ${ }^{19}{ }^{20}$ Moderate to severe patients with TBI may not have a preinjury baseline due to the unexpected nature of the injury.Studies without preinjury data will have their postinjury outcomes pooled within each timeframe defined as above and compared with controls. The sample size must have a minimum number of 30 individuals for each TBI severity group for better precision and confidence, ${ }^{4}$ to prevent selection bias that may be present in small studies. ${ }^{21}{ }^{22}$ Study designs of systematic reviews, meta-analyses, randomised controlled trials, casecontrol studies, cross-sectional studies and cohort studies published in peer-reviewed journals will be included. References from published systematic reviews and metaanalyses will be hand-searched for relevant articles. If the study is of an interventional nature, the study may be included should the control arm meet the inclusion criteria. Year of publication will span from 1988 to 2019. We chose the year of publication based on our objective to update the meta-analysis performed by Babikian and Asarnow. ${ }^{4}$ We also limited the earliest year of publication to 1988 (three decades) to limit heterogeneity given the changes in identification and management of TBI. ${ }^{4}$

Exclusion criteria include animal studies, non-traumatic acquired brain injuries, neurocognitive outcomes that do not fall into the domains chosen or small studies with a study population $<30$ children in each TBI severity group. Study designs of case reports and laboratory studies, 
publication types of commentaries, letters, books, newsletters, fact sheets, guidelines, editorials and any unpublished manuscripts will be excluded. Any studies not in the English language will be excluded.

\section{Information sources}

The literature search will cover the electronic databases: Medline (OVID interface), Embase (OVID interface), Cochrane Central Register of Controlled Trials (Wiley interface) and PsycINFO (OVID interface).

We will refer to the bibliography of included publications to ensure our literature search is adequately inclusive of relevant studies. We will also conduct searches of the authors to capture all relevant material that he or she has published. The bibliography of the included articles will be circulated to the systematic review team and paediatric TBI experts.

\section{Search strategy}

Qualitative and quantitative studies will be used. No restrictions, such as study design, will be imposed on the search. Medline, Embase, PsycINFO and Cochrane databases will be searched. Medical librarians have been consulted in drawing up a robust search strategy. The Medline strategy will be developed with input from the clinical review team. It will then be peer-reviewed by an expert in Search Strategy who is not otherwise associated with the project. Medical Subject Headings (MeSH) terms for Medline and Cochrane, and Emtree terms for Embase will be exploded as appropriate for each term's tree. Their synonyms will be included as title, abstract and keyword searches. The search terms pertaining to each aspect of the study are derived from various key articles found in preliminary searches. ${ }^{23-26}$

To ensure that there are no similar studies being carried out currently, the following electronic registries for trial protocols have been searched: PROSPERO, ClinicalTrials. gov, International Standard Randomised Controlled Trial Number registry, WHO International Clinical Trials Registry Platform and EU Clinical Trials Register.

The full search strategy can be found in online supplementary appendix 1. Strategic search terms include: population-paediatric, child, adolescent; exposure-TBI, head injury, traumatic brain haemorrhage; outcome-cognition, neurocognitive disorder, neuropsychology, executive function, perception, spatial navigation, psychomotor performance, memory, learning.

\section{Study records}

Literature search results will be uploaded to Covidence, ${ }^{27}$ an internet-based software program that facilitates collaboration among reviewers during the study selection process. Covidence follows the PRISMA 2009 guidelines and is our software of choice for this review. The studies will first be screened by title and abstract for relevancy. At this stage, only the PECO elements will be considered. During the second stage, the rest of the eligibility criteria will be appliedas the full texts are screened. Reasons for excluding each article will be recorded.

Two reviewers will independently screen the articles during both stages. Any conflicts will be resolved by a third independent reviewer, or by discussion. Neither of the review authors will be blind to the journal titles, study authors or institutions.

\section{Data management}

Data extraction will be done by two independent reviewers. Using a standardised form on Microsoft Excel, reviewers will extract the following from each article: study name, author(s), publication date, language, geographical origin, TBI definition, TBI severity as defined by GCS, study design, study phase, participant characteristics (eg, age, sex, comorbidity), neurocognitive domains investigated and their respective tests, and the time of each assessment in relation to injury. Whenever possible, primary quantitative data will be extracted for metaanalysis. The same information will also be extracted for the control group for the analysis. These controls include healthy children or children with non-neurological single-system injuries (eg, children with only orthopaedic injuries). If healthy and orthopaedic injury controls are present, we will preferentially select the healthy control. Subsequently, studies with healthy and orthopaedic injury controls will be combined. ${ }^{28}$ Controls that are classified otherwise will be excluded (eg, attention deficit hyperactivity disorder). Any qualitative data will be included as part of the narrative review.

The reviewers will extract data independently and in duplicate from each eligible study. To ensure consistency across reviewers, we will conduct training for all reviewers prior to the start of data extraction. We will do this by independent data extraction of separate reviewers on the same data sources followed by resolution of differences and development of a common understanding. Reviewers will resolve disagreements by discussion. We will contact study authors to resolve any uncertainties. A reminder will be sent to the author in 3 weeks. Should the author not reply within 1 month, we will consider the study excluded. ${ }^{29}$

\section{Outcomes and prioritisation}

Our primary outcome is to determine the progression of each neurocognitive domain over time as a result of mild, moderate and severe TBI. This will be done quantitatively, taking into account the age of the children at the time of injury (0-5 and $6-18$ years old) ${ }^{4}$ We will then perform a meta-regression by age if there are sufficient studies in both categories. The studies that use numerical tests to measure neurocognition will contribute to the meta-analysis. Studies will be presented and compared with controls where healthy, typically developing children will be preferentially picked over single-system orthopaedic patients. When preinjury data are present, we will perform a longitudinal design analysis. The remaining studies will be reviewed systematically and described. 
Studies that used previous DSM (DSM-III and DSM-IV) criteria for neurocognitive domains will be mapped to the current DSM-V criteria.

\section{Risk of bias individual studies}

The Scottish Intercollegiate Guidelines Network (SIGN) criteria $^{30}$ will be used for critical appraisal of the risk of bias (RoB) in each study. These quality assessment forms provide a systematic way of evaluating cross-study bias. An overall rating of high quality, acceptable, low quality or unacceptable will be given to each study. If there is insufficient detail reported in the study, the original study investigators will be contacted for more information. If there is still insufficient information available, we will report the RoB as 'unclear'. These judgements will be made independently by two reviewers based on the SIGN criteria. Disagreements will be resolved first by discussion and then by consulting a third reviewer for arbitration. We will consider each item in the RoB assessment independently without attempting to collate and assign an overall score.

\section{Data synthesis}

All assigned cognitive outcome measures will be analysed separately. Each cognitive domain will be pooled and stratified by TBI severity anddifferent time points.

Studies will be pooled using the DerSimonian and Laird method ${ }^{31} 32$ of inverse variance random effects standardised mean differences (SMD) between control and TBI severity groups. The pooled SMD with $95 \%$ CI will be presented in forest plots. Studies that report longterm respective outcomes longitudinally will be pooled by timepoint to account for within-subject correlation. Outcome estimates will be compared by TBI severity (mild vs moderate vs severe). For studies that report cognitive outcomes for preinjury and postinjury TBI groups, a separate analysis will be done, using paired SMDs to pool the studies.

For outcomes reported as median (IQR or range) instead of mean (SD), the median will be used to estimate the mean if the sample size is larger than 30 and there is no mention of the data being skewed. ${ }^{22}{ }^{33-35}$

Where there are missing data, the original authors will be contacted to obtain the data. If the missing data cannot be obtained, the study will be excluded from the meta-analysis but still included in the systematic review.

Data contributing to the systematic review will be presented in tables. A narrative synthesis will then be written about the different types of outcome measures used and how often these measures are used to measure the various cognitive domains over time.

If we have adequate studies, we will do an age-stratified subgroup analysis for each domain and timepoint.

\section{Meta-bias(es)}

To evaluate the presence of selective reporting of outcomes, we will look at the random effects model to assess the possibility of sample bias. A funnel plot ${ }^{36} 37$ will be used to further assess for publication bias.
Patient and public involvement

No patients involved.

\section{Confidence in cumulative evidence}

The strength of the cumulative evidence will be assessed at the final stage using the Grading of Recommendations Assessment, Development and Evaluation (GRADE). ${ }^{38}$ The evidence gathered will be assessed in the areas of risk of bias, precision and publication bias. Other areas will be considered when appropriate. The quality of evidence will be graded as high, moderate, low or very low.

\section{DISCUSSION}

\section{Ethics and dissemination}

This systematic review and meta-analysis aims to elucidate the true burden of paediatric TBI on neurocognition. The burden of paediatric TBI is increasing, as shown by rising hospital admission rates, ${ }^{39}$ and increasing costs. ${ }^{40}$ With improved trauma resuscitation and TBI management, mortality for severe TBI has decreased, but patients with both moderate and severe TBI often live with longterm neurological sequelae. ${ }^{41}$ There is an urgent need to accurately define the devastating effects of paediatric TBI, especially those with moderate and severe TBI on short-term and long-term neurocognition. Children with mild TBI often have good recovery, nevertheless, their neurocognitive outcomes over time deserve study and proper documentation.

A systematic appraisal is needed to understand these long-term neurocognitive deficits. Current limitations to individual studies include a great variability in the TBI severity, definition of neurocognitive deficits and outcome measures used. For example, a 12-month longitudinal study assessed attention, working memory and processing speed in moderate and severe TBI only, ${ }^{10}$ while another 12-month study based in Los Angeles, US studied language, memory, psychomotor function and inhibition in mild TBI. ${ }^{7}$ Another study covered all TBI severities and spanned 10 years but assessed domains of intelligence, adaptive behaviour, executive function and social function. ${ }^{11}$ Individual studies, while informative in their respective domains, do not aid in the overall understanding of the neurocognitive outcomes for this vulnerable paediatric population. The increasing and sophisticated measures used to assess neurocognition in the past decade present a golden opportunity to consolidate short-term and long-term neurocognitive outcomes.

The strength of this study lies in the methodology, which makes it possible to capture a wide range of data on clearly defined neurocognitive outcomes as well as to stratify these outcomes based on TBI severity and time point in order to perform a comprehensive analysis of each domain. Our protocol is registered with PROSPERO and is designed with best practice methods. By comparing each TBI severity group to a control group within each study, and then aggregating the results across studies, 
we will better positioned to understand the trajectory of neurocognitive changes over time.

With the results of our study, we will be able to understand the true burden of TBI on a much larger scale. This study has the potential to define the permanent and life-changing consequences that are more often found in moderate and severe paediatric TBI, to inform decisions made by policy-makers and government agencies. With a clearer understanding of specific neurodevelopmental deficits thatchildren with TBI suffer from, early intervention programmes can be tailored to improve the child's developmental trajectory. To care for these injured children in the long-run, targeted educational and vocational opportunities need to be created and sustained.

This study will also enable us to understand how different neurocognitive scales are being used in various parts of the world. It can provide valuable data to dedicated TBI work groups when making future decisions on which common data elements are relevant and important for TBI research. Finally, by consolidating the current published literature on the topic, we hope to guide future researchers by providing critical knowledge on long-term neurocognitive outcomes that can be used to assess the effectiveness of interventional strategies for TBI.

Ethics approval is not required for this study.

\section{Limitations}

The search terms in the strategy are explored in the $\mathrm{MeSH}$ browser. It provides a systematic and standardised way of searching, however, it may not fully cover all the articles relevant to the research question if they are not indexed under the MeSH terms used. For example, there is no MeSH term directly related to 'social cognition', which is one of the key neurocognitive domains used in this paper. In addition, the limited number of databases employed and restrictions to English increase the possibility of missing out relevant studies.

Having predetermined the selected domains to be reviewed, we are not able to include the studies of outcomes that are out of our selection but still relevant to the issue. One example is a study on social aggression following TBI, ${ }^{42}$ which although may not directly affect cognition, still plays a role in a patient's ability to make decisions, ${ }^{43}$ and affects social functioning which leads to consequences in all areas of life. There is room for further review and study of these factors that contribute to patients' outcomes.

We will collect information on important prognostic factors (eg, age at time of injury and the mechanism of injury) and present this as part of the systematic review. However, we recognise that not all variables will be used to stratify the neurocognitive outcomes at the meta-analysis and will give priority to the severity of TBI and the time since injury.

Children who suffer from TBI, in particular moderate to severe TBI, suffer from neurocognitive deficits. This systematic review and meta-analysis aims to provide a comprehensive landscape of the true burden of paediatric
TBI across neurocognitive domains with the passage of time.

Author affiliations

${ }^{1}$ Lee Kong Chian School of Medicine, Singapore

${ }^{2}$ Duke-NUS Medical School, Singapore

${ }^{3}$ Department of Emergency Medicine, KK Women's and Children's Hospital, Singapore

${ }^{4}$ NUS Yong Loo Lin School of Medicine, Singapore

${ }^{5}$ Centre for Quantitative Medicine, Duke-NUS Medical School, Singapore

${ }^{6}$ Children's Intensive Care Unit, KK Women's and Children's Hospital, Duke-NUS

Medical School, Singapore

${ }^{7}$ Department of Emergency Medicine, KK Women's and Children's Hospital, DukeNUS Medical School, Singapore

Contributors MSLG and S-LC coordinated the study. DSHL, MSLG and S-LC developed the search strategy and registered the protocol. DSHL drafted the manuscript. DSHL, JLG, MSLG, SSMG, RS, S-LC and JHL helped to inform the study design and revise the manuscript. MSLG obtained funding for the review. RS developed the meta-analytical strategy. All authors read and approved the final manuscript.

Funding This review is financially supported by Academic Medicine - Enhancing Training Healthcare Outcomes \& Standards (ETHOS) Duke-NUS Medical Student Fellowship (AY2019-AY2020). Duke-NUS was not involved in the design of the protocol and analysis plan of the review, and will not provide input on the interpretation of the results.

Competing interests None declared.

Patient and public involvement Patients and/or the public were involved in the design, or conduct, or reporting, or dissemination plans of this research. Refer to the Methods section for further details.

Patient consent for publication Not required.

Provenance and peer review Not commissioned; externally peer reviewed.

Open access This is an open access article distributed in accordance with the Creative Commons Attribution Non Commercial (CC BY-NC 4.0) license, which permits others to distribute, remix, adapt, build upon this work non-commercially, and license their derivative works on different terms, provided the original work is properly cited, appropriate credit is given, any changes made indicated, and the use is non-commercial. See: http://creativecommons.org/licenses/by-nc/4.0/.

ORCID iDs

Mark Sen Liang Goh http://orcid.org/0000-0003-1183-314X

Shu-Ling Chong http://orcid.org/0000-0003-4647-0019

\section{REFERENCES}

1 Dewan MC, Mummareddy N, Wellons JC, et al. Epidemiology of global pediatric traumatic brain injury: qualitative review. World Neurosurg 2016;91:497-509.

2 Zaloshnja E, Miller T, Langlois JA, et al. Prevalence of long-term disability from traumatic brain injury in the civilian population of the United States, 2005. J Head Trauma Rehabil 2008;23:394-400.

3 Centers for Disease Control and Prevention. Report to Congress on traumatic brain injury in the United States: epidemiology and rehabilitation. Atlanta, GA: National Center for Injury Prevention and Control; Division of Unintentional Injury Prevention, 2005.

4 Babikian T, Asarnow R. Neurocognitive outcomes and recovery after pediatric TBI: meta-analytic review of the literature. Neuropsychology 2009;23:283-96.

5 Anderson V, Brown S, Newitt $\mathrm{H}$, et al. Educational, vocational, psychosocial, and quality-of-life outcomes for adult survivors of childhood traumatic brain injury. J Head Trauma Rehabil 2009;24:303-12.

6 Balaban T, Hyde N, Colantonio A. The effects of traumatic brain injury during adolescence on career plans and outcomes. Phys Occup Ther Pediatr 2009;29:367-83.

7 Babikian T, Satz P, Zaucha K, et al. The UCLA longitudinal study of neurocognitive outcomes following mild pediatric traumatic brain injury. J Int Neuropsychol Soc 2011;17:886-95.

8 Bardoni A, Galbiati S, Recla M, et al. Evolution of the cognitive profile in school-aged patients with severe TBI during the first 2 years of neurorehabilitation. Brain Inj 2013;27:1395-401. 
9 McKinlay A, Grace RC, Horwood LJ, et al. Long-Term behavioural outcomes of pre-school mild traumatic brain injury. Child Care Health Dev 2010;36:22-30.

10 Pivonka-Jones J, Johnson V, Freier Randall K, et al. C-36 * pediatric traumatic brain injury: longitudinal neurocognitive outcomes. Archives of Clinical Neuropsychology 2014;29:586-7.

11 Anderson V, Godfrey C, Rosenfeld JV, et al. Predictors of cognitive function and recovery 10 years after traumatic brain injury in young children. Pediatrics 2012;129:e254-61.

12 Beauchamp M, Catroppa C, Godfrey C, et al. Selective changes in executive functioning ten years after severe childhood traumatic brain injury. Dev Neuropsychol 2011;36:578-95.

13 American Psychiatric Association. DSM-5 Task Force. In: Diagnostic and statistical manual of mental disorders: DSM-5 ${ }^{\mathrm{TM}}$. 5th. Arlington, VA, US: American Psychiatric Publishing, Inc, 2013.

14 Morgan RL, Whaley P, Thayer KA, et al. Identifying the PECO: a framework for Formulating good questions to explore the association of environmental and other exposures with health outcomes. Environ Int 2018;121:1027-31.

15 Moher D, Shamseer L, Clarke M, et al. Preferred reporting items for systematic review and meta-analysis protocols (PRISMA-P) 2015 statement. Syst Rev 2015;4:1.

16 Hardin AP, Hackell JM, Committee on practice and ambulatory medicine. Age limit of pediatrics. Pediatrics 2017;140:e20172151.

17 Teasdale G, Jennett B. Assessment of coma and impaired consciousness. The Lancet 1974;304:81-4.

18 Menon DK, Schwab K, Wright DW, et al. Position statement: definition of traumatic brain injury. Arch Phys Med Rehabil 2010;91:1637-40.

19 Bleiberg J, Cernich AN, Cameron K, et al. Duration of cognitive impairment after sports concussion. Neurosurgery 2004;54:1073-80.

20 Covassin T, Elbin RJ, Nakayama Y. Tracking neurocognitive performance following concussion in high school athletes. Phys Sportsmed 2010;38:87-93.

21 Dechartres A, Trinquart L, Boutron I, et al. Influence of trial sample size on treatment effect estimates: meta-epidemiological study. BMJ 2013;346:f2304.

22 Hozo SP, Djulbegovic B, Hozo I. Estimating the mean and variance from the median, range, and the size of a sample. BMC Med Res Methodol 2005;5:13.

23 Lumba-Brown A, Yeates KO, Sarmiento K, et al. Diagnosis and management of mild traumatic brain injury in children: a systematic review. JAMA Pediatr 2018;172:e182847

24 Kennedy E, Cohen M, Munafò M. Childhood traumatic brain injury and the associations with risk behavior in adolescence and young adulthood: a systematic review. J Head Trauma Rehabil 2017;32:425-32.

25 D'Souza A, Mollayeva S, Pacheco N, et al. Measuring change over time: a systematic review of Evaluative measures of cognitive functioning in traumatic brain injury. Front Neurol 2019;10:353.
26 Karr JE, Areshenkoff CN, Garcia-Barrera MA. The neuropsychological outcomes of concussion: a systematic review of meta-analyses on the cognitive sequelae of mild traumatic brain injury. Neuropsychology 2014;28:321-36.

27 Covidence. Covidence systematic review software.Melbourne, Australia: veritas health innovation, 2019. Available: www.covidence. org

28 Mathias JL, Dennington V, Bowden SC, et al. Community versus orthopaedic controls in traumatic brain injury research: how comparable are they? Brain Inj 2013;27:887-95.

29 Guevara J, Keren R, Nihtianova S, et al. How do authors respond to written requests for additional information?. XIII Cochrane Colloquium 2005 OCT 22-26. Melbourne, Australia, 2005;

30 Sign. Checklists and notes, 2019. Available: https://www.sign.ac.uk/ checklists-and-notes.html [Accessed 13 Sep 2019].

31 DerSimonian R, Laird N. Meta-Analysis in clinical trials. Control Clin Trials 1986;7:177-88.

32 DerSimonian R, Laird N. Meta-Analysis in clinical trials revisited. Contemp Clin Trials 2015;45:139-45.

33 Higgins JPTThomas J, Chandler J, eds. Cochrane Handbook for systematic reviews of interventions. version 6.0. Cochrane, 2019. www.training.cochrane.org/handbook

34 Wan X, Wang W, Liu J, et al. Estimating the sample mean and standard deviation from the sample size, median, range and/or interquartile range. BMC Med Res Methodol 2014;14:135.

35 Luo D, Wan X, Liu J, et al. Optimally estimating the sample mean from the sample size, median, mid-range, and/or mid-quartile range. Stat Methods Med Res 2018;27:1785-805.

36 Egger M, Davey Smith G, Schneider M, et al. Bias in meta-analysis detected by a simple, graphical test. BMJ 1997;315:629-34.

37 Sterne JAC, Egger M. Funnel plots for detecting bias in metaanalysis. J Clin Epidemiol 2001;54:1046-55.

38 Guyatt GH, Oxman AD, Vist GE, et al. Grade: an emerging consensus on rating quality of evidence and strength of recommendations. BMJ 2008;336:924-6.

39 Chen C, Peng J, Sribnick E, et al. Trend of age-adjusted rates of pediatric traumatic brain injury in U.S. emergency departments from 2006 to 2013. Int J Environ Res Public Health 2018;15:1171.

40 Graves JM, Rivara FP, Vavilala MS. Health care costs 1 year after pediatric traumatic brain injury. Am J Public Health 2015;105:e35-41.

41 Popernack ML, Gray N, Reuter-Rice K. Moderate-To-Severe traumatic brain injury in children: complications and rehabilitation strategies. J Pediatr Health Care 2015;29:e1-7.

42 Cole WR, Gerring JP, Gray RM, et al. Prevalence of aggressive behaviour after severe paediatric traumatic brain injury. Brain Inj 2008;22:932-9.

43 Kuin N, Masthoff E, Kramer M, et al. The role of risky decisionmaking in aggression: a systematic review. Aggress Violent Behav 2015;25:159-72. 